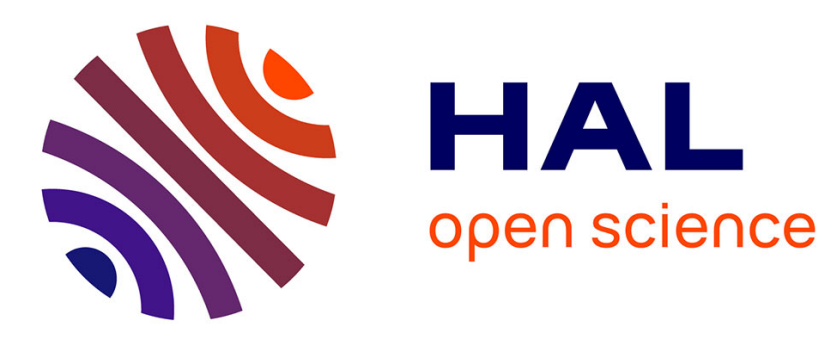

\title{
Out in Africa. Same Sex Desire in Sub Saharan Literatures and Cultures \\ Larissa Kojoué
}

\section{To cite this version:}

Larissa Kojoué. Out in Africa. Same Sex Desire in Sub Saharan Literatures and Cultures. Canadian Journal of African Studies / La Revue canadienne des études africaines, 2016, pp.130 - 132. 10.1080/00083968.2016.1145383 . inserm-01876173

\section{HAL Id: inserm-01876173 https://www.hal.inserm.fr/inserm-01876173}

Submitted on 18 Sep 2018

HAL is a multi-disciplinary open access archive for the deposit and dissemination of scientific research documents, whether they are published or not. The documents may come from teaching and research institutions in France or abroad, or from public or private research centers.
L'archive ouverte pluridisciplinaire HAL, est destinée au dépôt et à la diffusion de documents scientifiques de niveau recherche, publiés ou non, émanant des établissements d'enseignement et de recherche français ou étrangers, des laboratoires publics ou privés. 


\section{Compte rendu de lecture}

\section{Larissa Kojoué}

To cite this article: Larissa Kojoué (2016): Compte rendu de lecture, Canadian Journal of African Studies / Revue canadienne des études africaines

To link to this article: http://dx.doi.org/10.1080/00083968.2016.1145383

\section{Published online: 07 Mar 2016.}

Submit your article to this journal $\sqsubset$

Q View related articles ¿

View Crossmark data \lceil 
BOOK REVIEW

\title{
Out in Africa: Same-Sex Desire in Sub-Saharan Literatures and Cultures, by
}

\author{
Chantal Zabus, Suffolk: Boydell \& Brewer, 2013, x 298 pp.
}

Out in Africa plonge le lecteur dans les rayons d'une immense bibliothèque consacrée aux écritures du désir et de la sexualité entre personnes de même sexe en Afrique subsaharienne. Cette initiative ambitieuse rassemble pas moins d'une cinquantaine d'auteurs africains originaires de I'Afrique de l'Ouest, de l'Afrique centrale, de l'Afrique de l'Est et de l'Afrique australe, autour d'un répertoire impressionnant de nouvelles, de romans, de poèmes, d'autobiographies et de films allant de 1870 à 2010. Ces littératures mettent en scène le désir homosexuel, l'attirance mutuelle (ou non) entre hommes et entre femmes.

En pénétrant dans cette bibliothèque, on trouve en tête de gondole des références anthropologiques datant de la période précoloniale et coloniale (chapitre 1). Ces références, essentiellement européennes, concernent le caractère situationnel des relations homosexuelles. Les Européens qui arrivent en Afrique sont autorisés à entretenir des rapports homosexuels avec des hommes africains efféminés, plutôt qu'avec les femmes autochtones considérées comme frigides et malodorantes. Certaines pratiques traditionnelles comme les "female husbands" chez les Igbo du Nigéria ou les Kikuyu du Kenya, les "mines mariages "chez les travailleurs sud-africains entre des "boss-boys " et des "boy-wives " sont aussi documentées, lesquelles ont favorisé une importation du vocabulaire sexuel européen. Dans la suite de ces discours sur l'homosexualité en Afrique, Chantal Zabus a choisi deux textes pionniers d'une forte dimension érotique qui racontent sans les nommer, des relations entre des colons avec leurs pages africains (chapitre 2). Il s'agit de Le roman d'un Spahi (1881) du français Pierre Loti, et de My Kalulu, prince, king, and slave (1873) de l'explorateur anglais Henry Stanley.

C'est à partir du chapitre 3 qu'on commence véritablement à prendre plaisir à lire Out in Africa. On découvre enfin comment les écrivains africains représentent le désir homosexuel. L'Occident, à travers sa religion, surtout l'église (mission catholique) et la formation des élites pour consolider l'État naissant au lendemain des indépendances est considéré comme responsable de l'exposition et de l'initiation des jeunes africains aux pratiques homosexuelles. C'est ce qu'on retrouve dans les premiers textes qui y font référence: The Interpreters (1963) du nigérian Wole Soyinka; Le devoir de violence (1968) du malien Yambo Ouologuem; A few nights and days (1970) du camerounais Mbella Sonne Dipoko; No past, no present, no future (1973) du sierra léonais Yulisa Amadu Maddy; Why are we so blest? (1974) ainsi que Two thousand seasons (1979) du ghanéen Kwei Armah.

Cette représentation de l'homosexualité comme une importation voire une pathologie imprègne aussi les textes des écrivaines abordant sur la sexualité féminine. Plus récents et moins nombreux que leur contrepartie masculine, les premiers textes présentent la sexualité entre femmes comme un acte non réciproque, non consensuel et voué à l'échec. C'est le cas de Ripples in the pool (1975) de la kenyanne Rebeka Njau; Our sister killjoy (1977) de la ghanéene Ama Ata Aidoo. Ces représentations des désirs homosexuels demeurent fortes dans l'imaginaire populaire africain aujourd'hui, bien que l'écriture de la sexualité ait évolué depuis le milieu des années 1990. Les écrivains africains vont progressivement s'émanciper de cette vision extra centrée, moralisée et pathologisée de l'homosexualité pour l'aborder sous un angle plus humanisé, celui de l'amour, du plaisir, voire de l'identité. On peut le constater dans les chapitres 4 et 5 . Qu'ils soient décrits dans un style gothique ou fantastique, lyrique, autobiographique ou polémique, ces désirs sont cependant toujours contrariés soit par la race, la loi, l'âge, la religion, soit par la folie d'un 
partenaire, l'exil, le poids des conventions auxquelles certains écrivains semblent adhérer. Au rang de cette nouvelle écriture de la sexualité entre femmes, on peut citer: Sky-high flames (2005) de Unomah Azuah; Jambula tree (2006) de Monica Arac de Nyeko; Jumping Monkey Hill (2009) de Chimamanda Ngozi Adichie; White is for witching (2009) de Helen Oyeyemi; ou encore The world unseen (2001) de Shamim Sarif. The smell of apples (1995) de Marc Behr, issu du même registre que Time of our darkness (1989) de Stephen Gray, marquent inévitablement une rupture avec la représentation de l'homosexualité masculine. Sous la plume de ces auteurs blancs sud-africains, l'amour entre hommes défie l'oppression de l'apartheid et transcende les barrières législatives, traditionnelles et culturelles.

L'écriture de la sexualité entre personnes de même sexe est aussi très présente dans des récits métaphysiques africains comme on peut le voir au chapitre 6. Des écrivaines comme Calixte Beyala (Femme nue, femme noire 2003), K. Sello Duiker (The quiet violence of dreams 2001), Doumbi Fakoly (La révolte des galsénésiennes 1994) ou Nkundi Zandile Nkabinde (Black bull, ancestors and me 2008) ont recours à la figure des esprits possédant des pouvoirs surnaturels pour justifier les relations homosexuelles. Ce recours aux mythes, très présent dans l'imaginaire africain et dans l'écriture féminine, révèle la complexité des relations homosexuelles entre femmes. Celles-ci relèveraient du sublime, comme pour justifier la profondeur des sentiments ainsi exprimés. Dans son chapitre conclusif, Chantal Zabus analyse la question "trans" en Afrique en la replaçant dans une dynamique globale, une position qu'elle exprime en opposition à un "apartheid du sexe".

La manière dont le désir homosexuel est représenté dans les textes écrits ou vidéos des auteurs africains est un signe de l'émancipation de l'écriture de la sexualité en contextes africains. Loin d'être un simple caprice ou un effet de mode, cela indique une volonté d'écrire ce que les convenances veulent taire ou étouffer, le souhait de créer des espaces de liberté pour des personnes dont l'identité est niée, désavouée et condamnée. Quoique non militante (pour la plupart), ces écritures de la sexualité manifestent le souci de tenir compte des transformations qui traversent la société globale. Elles contribuent ainsi à démystifier le sexe et l'homosexualité en Afrique, sans pour autant en faire un objet banal ou acceptable, ni le détacher complètement d'une vision occidentalo-centrée, comme en témoigne le nombre de textes rédigés par les écrivains de la diaspora, et le nombre d'histoires se terminant dans la douleur.

En parcourant les rayons de cette bibliothèque virtuelle, le chercheur, l'enseignant, l'étudiant ou le lecteur francophone risque cependant de rester sur sa faim. A l'évidence, les références littéraires de langue anglaise sont surreprésentées. Cela est révélateur du déséquilibre des sources linguistiques et géographiques dans le choix de Chantal Zabus. Manquent ainsi à l'appel des œuvres francophones incontournables comme celles d'Angèle Rawiri (Fureurs et cris de femme 1989), Frieda Ekotto (Chuchote pas trop 2006, Portrait d'une jeune artiste de Mbona Mbella 2008) qui racontent des relations explicites, ouvertes et assumées entre femmes! On pense aussi à Sami Tchak (La fête des masques 2004) ou encore Bertrand Nguyen Matoko (Le flamant noir 2004) qui n'usent d'aucun détour ni stratagème pour parler d'attirance mutuelle entre hommes, et cela dans une perspective non misérabiliste. Et pourquoi ne pas mentionner la dimension érotique bien que non structurante des relations entre femmes dans les romans de Ken Bugul (Riwan ou le chemin de sable, 1999; De l'autre côté du regard, 2003) ou Rue Félix-Faure (2005) ou celui de Calixte Beyala (C'est le soleil qui m'a brûlée, 1987 et Tu t'appelleras Tanga, 1988)? Si les trois quart de références sont d'expression anglaise, l'auteur ne rend pas justice aux écrivain(e)s africain(e)s et aux lecteurs d'expression française dont elle est pourtant proche (Chantal Zabus vit et travaille en France).

Londres, Paris et New York sont les principaux lieux d'édition des 142 sources primaires recensées (pour une bibliographie impressionnante de critiques littéraires, de références anthropologiques, historiques, sociologiques et philosophiques). Seule une douzaine des sources primaires citées se trouve en Afrique, et essentiellement en Afrique du Sud (Pretoria et Johannesburg). Quelques publications ont été éditées au Nigéria (Lagos, Ibadan). La question du lieu de publication, de la distance avec le contexte de ce qu'on écrit se pose alors: lorsqu'on publie dans un contexte 
différent de celui de la production, les horizons d'attentes peuvent être complètement différents et générer des malentendus quant au sens des réalités exprimées. Pourquoi la quasi-totalité des références sur la sexualité entre hommes et la sexualité entre femmes sont-elles publiées en terrains non africains? Ce choix ne renforce-t-il pas l'extranéité des pratiques homosexuelles en Afrique subsaharienne? A moins qu'il ne légitime la marginalité de l'écriture de l'homosexualité chez les écrivains africains vivant en Afrique?

La mise en visibilité de la question homosexuelle par les écrivains africains telle que présentée par Chantal Zabus ouvre des perspectives immenses d'études sur l'évolution des discours sur le genre et la sexualité en Afrique. Out in Africa est un peu trop optimiste quant à l'évolution des représentations de l'homosexualité sur le continent. En témoignent le durcissement des sanctions concernant les personnes homosexuelles et les frustrations postcoloniales qui semblent s'y cristalliser. Cependant, parce qu'elles participent de l'historicité du continent, les littératures subsahariennes de ces deux dernières décennies dessinent d'autres possibles: les écrivains africains d'aujourd'hui, tout comme ceux d'hier, sont des acteurs idéologiques du changement de la société. 